\title{
A força de atrito estático não é conservativa
}

\author{
The force of static friction is not conservative
}

\author{
Dêivid Rodrigo da Silva ${ }^{1}$, Paulo Peixoto*1 \\ ${ }^{1}$ Núcleo de Formação Docente, Universidade Federal de Pernambuco, Caruaru, PE, Brasil
}

Recebido em 01 de Maio de 2017. Aceito em 05 de Junho de 2017

\begin{abstract}
Neste artigo mostramos que, em uma abordagem macroscópica, a força de atrito estático não pode ser classificada como conservativa.

Palavras-chave: força de atrito estático, forças conservativas, teorema trabalho - energia cinética, rolamento.
\end{abstract}

In this paper we show that, in a macroscopic approach, the force of static friction cannot be classified as conservative.

Keywords: force of static friction, conservative forces, work - kinetic energy theorem, rolling motion.

\section{Introdução}

Em um trabalho publicado em 2003 [1], Silva e colaboradores afirmaram: "Este artigo propõe um experimento visando demonstrar que a força de atrito estático é conservativa". Para isso, abandonaram em repouso uma esfera no topo de um plano inclinado e verificaram que, para inclinações não muito acentuadas (garantindo, assim, que a esfera rolasse sem deslizamento), e dentro do erro experimental, a energia mecânica da esfera no início e ao final do plano é a mesma. Daí concluíram que a força de atrito estático - uma das forças que agem sobre a esfera, e sem a qual não haveria rolamento - é uma força conservativa.

À primeira vista, pode parecer uma conclusão correta, mas houve um erro de interpretação. Indo direto ao ponto: no rolamento sem deslizamento de uma esfera ideal ao longo de um plano inclinado ideal, a força de atrito estático não realiza trabalho - já que, a cada instante, age sempre em um ponto em repouso (o ponto da esfera em contato com o plano inclinado, naquele instante). Uma força que não realiza trabalho sobre um corpo não pode alterar sua energia mecânica, é claro, mas de forma alguma isso significa que ela seja uma força conservativa.

Na próxima seção mostraremos, através de um exemplo muito simples, que a força de atrito estático não pode ser classificada - em uma abordagem macroscópica - como uma força conservativa. Na seção subsequente, faremos uma análise teórica do rolamento de uma esfera ideal sobre um plano inclinado ideal, no que concerne à conservação da energia e ao papel desempenhado pela força de atrito estático.

Estamos admitindo que os leitores deste artigo têm bom conhecimento de mecânica newtoniana ao nível tipicamente trabalhado no primeiro ano de um curso de graduação em física ou engenharia [2 8].

*Endereço de correspondência: phrpeixoto@yahoo.com.br

\section{Forças conservativas e a força de atrito estático}

Uma força $\mathbf{F}$ é classificada como conservativa (i) se é função apenas da posição $\mathbf{r}$ da partícula sobre a qual atua e (ii) se o trabalho dessa força entre dois pontos quaisquer 1 e 2 independe da trajetória da partícula entre os mesmos - ou, equivalentemente, se o trabalho de $\mathbf{F}$ ao longo de qualquer caminho fechado é nulo. Com essas duas condições satisfeitas, podemos associar a $\mathbf{F}(\mathbf{r})$ uma função escalar $U(\mathbf{r})$, denominada energia potencial, tal que $\mathbf{F}=-\nabla U$ e - se a força resultante sobre a partícula é igual a $\mathbf{F}$ - a soma $E \equiv T+U, \operatorname{com} T \equiv$ $m v^{2} / 2$, é constante, sendo $m$ a massa da partícula e $v$ o módulo de sua velocidade (que em geral não é constante). Como você sabe, as quantidades $T$ e $E$ são denominadas, respectivamente, energia cinética e energia mecânica. (Para detalhes e aprofundamento, uma opção é o estudo do capítulo 4 do livro de mecânica clássica do Taylor [9].)

Para mostrar que a força de atrito estático não pode ser classificada - em uma abordagem macroscópica ${ }^{1}$ como uma força conservativa, é suficiente apresentar um exemplo em que uma das duas condições (i) e (ii), acima, não é satisfeita.

A Fig.11 ilustra um sistema de dois blocos oscilando amortecidamente pela ação combinada da força exercida por uma mola e da força de atrito cinético exercida pelo piso. O bloco B acompanha o movimento do bloco $\mathrm{A}$ devido à ação da força de atrito estático, que denotaremos por $\mathbf{f}_{\mathbf{e}}$, e que é a força resultante sobre B. Considere dois instantes $t_{1}$ e $t_{2}>t_{1}$ em que os blocos passam pela posição de equilíbrio (quando o mola está relaxada, ou seja, nem comprimida, nem distendida). Devido ao atrito entre o bloco A e o piso, a velocidade dos blocos no

\footnotetext{
${ }^{1}$ Uma análise microscópica do atrito não faz parte do escopo deste artigo.
} 


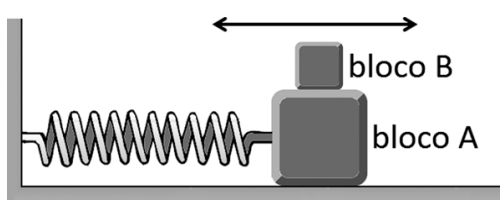

Figura 1: Blocos oscilando amortecidamente, devido ao atrito cinético entre o bloco $A$ e o piso. $O$ bloco $B$ acompanha $o$ movimento do bloco A pela ação da força de atrito estático.

instante $t_{2}$ é menor que no instante $t_{1}$. Assim, aplicando o teorema trabalho - energia cinética 2 a à força $\mathbf{f}_{\mathbf{e}}$ agindo no bloco B (modelado como uma partícula) entre os instantes $t_{1}$ e $t_{2}$, obtemos um trabalho de $\mathbf{f}_{\mathbf{e}}$ não-nulo em um caminho fechado - o que nos permite concluir que a força de atrito estático não pode ser classificada como conservativa.

\section{Rolamento de uma esfera sobre um plano inclinado: conservação da energia e o papel da força de atrito estático}

Se a força de atrito estático não pode ser classificada como conservativa, é correto dizer que um corpo sob sua ação não pode ter sua energia mecânica conservada? Não, não é correto. Veremos isso analisando o rolamento de uma esfera maciça ideal de massa $M$ e raio $R$ abandonada em estado de repouso em um plano com inclinação $\theta$, a uma altura inicial $h_{0}$ medida da base do plano até o centro de massa da esfera (Fig.2).

Modelaremos a esfera como um sistema de partículas de massa total $M$ e aplicaremos à mesma o teorema trabalho - energia cinética,

$$
W_{\text {total }}=\Delta T,
$$

em que $\Delta T$ é a variação da energia cinética do sistema entre dois estados 1 e 2 e $W_{\text {total }}$ é a soma dos trabalhos de todas as forças (internas e externas) que atuam sobre as partículas do sistema na evolução do estado 1 ao estado 2.

Podemos convenientemente expressar (veja, por exemplo, o início da seção 9.5 do Thornton-Marion [10]):

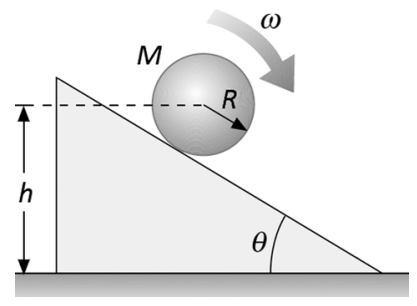

Figura 2: Rolamento de uma esfera ideal sobre um plano inclinado ideal fixo. A esfera é abandonada em estado de repouso à altura inicial $h=h_{0}$.
$T=\frac{1}{2} M v_{\mathrm{cm}}^{2}+T_{\text {rel }}$, em que $v_{\mathrm{cm}}$ é o módulo da velocidade do centro de massa do sistema (no referencial do laboratório) e $T_{\text {rel }}$ é a soma das energias cinéticas das partículas do sistema em relação ao centro de massa. No caso da esfera da Fig.2, temos $v_{\mathrm{cm}}=\omega R$ (condição de rolamento sem deslizamento) e $T_{\text {rel }}=\frac{1}{2} I \omega^{2}$, em que $I=\frac{2}{5} M R^{2}$ é o momento de inércia da esfera (maciça) e $\omega$ é sua velocidade angular - ambos em relação ao eixo de rotação (que intercepta o centro de massa). Reunindo tudo, e lembrando que a esfera parte do repouso, podemos expressar a variação da energia cinética da esfera em função do módulo da velocidade de seu centro de massa:

$$
\Delta T=\frac{7}{10} M v_{\mathrm{cm}}^{2}
$$

O cálculo de $W_{\text {total }}$ desempenha papel central nesta discussão. No caso de um corpo rígido (e a esfera na Fig. 2 é um corpo rígido), o trabalho total das forças internas entre dois estados quaisquer é nulo (veja Apêndice). As forças externas que agem sobre a esfera são a força

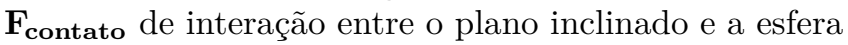
e os pesos das partículas que constituem esse sistema.

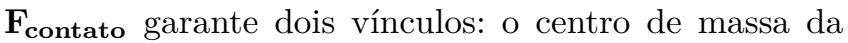
esfera move-se paralelamente ao plano inclinado, e o rolamento se dá sem deslizamento $\left(v_{\mathrm{cm}}=\omega R\right)$. Mais especificamente, o primeiro vínculo é garantido pela componente de $\mathbf{F}_{\text {contato }}$ perpendicular ao plano inclinado, $\mathbf{f}_{\mathbf{n}}$ (que chamamos de força normal), enquanto o segundo vínculo resulta da ação da componente de $\mathbf{F}_{\text {contato }}$ paralela ao plano inclinado, $\mathbf{f}_{\mathbf{e}}$ (que é a força de atrito estático) ${ }^{2}$ Pois bem, voltando ao cálculo de $W_{\text {total }}$, temos que $\mathbf{f}_{\mathbf{n}}$ e $\mathbf{f}_{\mathbf{e}}$ (e $\mathbf{F}_{\text {contato }}$, como um todo) não realizam trabalho, porque, a cada instante, agem sempre em um ponto em repouso: o ponto da esfera em contato com o plano inclinado, naquele instante. Isso precisa ser bem compreendido. Embora a esfera esteja em movimento, estamos modelando-a como um sistema de partículas, e as forças $\mathbf{f}_{\mathbf{n}}$ e $\mathbf{f}_{\mathbf{e}}$ agem sempre sobre uma partícula em repouso, não sobre a esfera como um todo. Por isso, o trabalho de cada uma dessas duas forças é nulo. Assim, $W_{\text {total }}$ é igual à soma dos trabalhos dos pesos das partículas que constituem a esfera. Trabalhemos com um sistema discreto de $N$ partículas, de massas $m_{1}, m_{2}, \ldots, m_{N}{ }^{3}$ Do estado inicial de repouso da esfera ( $h=h_{0}$ na Fig. 2) a um estado em que seu centro de massa se encontra a uma altura $h\left(<h_{0}\right)$ da base do plano inclinado, a partícula de massa $m_{i}(i \in\{1,2, \ldots, N\})$ sofre uma variação de altura $\Delta h_{i}$, e, assim, o trabalho realizado por seu peso é $W_{P_{i}}=-m_{i} g \Delta h_{i}$. Segue que $W_{\text {total }}=\sum_{i=1}^{N} W_{P_{i}}=$

\footnotetext{
Sabemos que há um valor máximo para a força de atrito estático, mas estamos supondo aqui que há atrito suficiente para que o rolamento se dê sem deslizamento. Em termos experimentais, isso significa que há um valor máximo para a inclinação $\theta$ do plano com a qual podemos trabalhar. (Veja, por exemplo a seção 5 do artigo de Silva e colaboradores 1. .)

${ }^{3} \mathrm{~A}$ transição para um sistema contínuo - mais adequado à modelagem de uma esfera - é trivial: basta substituir os somatórios por integrais, e usar quantidades infinitesimais apropriadas. Mas o resultado para o cálculo que faremos seria o mesmo.
} 
$-g \sum_{i=1}^{N} m_{i} \Delta h_{i}=-g \Delta\left(\sum_{i=1}^{N} m_{i} h_{i}\right)$. De acordo com a definição de centro de massa de um sistema de partículas de massa total $M$, e lembrando que $h$ é a altura do centro de massa da esfera, temos $\sum_{i=1}^{N} m_{i} h_{i}=M h$, e, portanto, $W_{\text {total }}=-M g \Delta h$. Como $\Delta h=h-h_{0}<0$, podemos substituir $-\Delta h$ por $|\Delta h|$, obtendo

$$
W_{\text {total }}=M g|\Delta h| \text {. }
$$

Substituindo (2) e (3) em (1), obtemos:

$$
M g|\Delta h|=\frac{7}{10} M v_{\mathrm{cm}}^{2} .
$$

No trabalho publicado em 2003 [1], Silva e colaboradores submeteram a igualdade (4), acima, a um teste experimental 4 e verificaram que, para inclinações não muito acentuadas (garantindo, assim, que a esfera rolasse sem deslizamento), e dentro do erro experimental, a igualdade (4) é válida. O problema é que daí concluíram que a força de atrito estático - uma das forças que agem sobre a esfera, e sem a qual não haveria rolamento - é uma força conservativa.

No decurso para obtenção da igualdade (4), mostramos, de forma totalmente consistente com a mecânica newtoniana, que a força de atrito estático sobre uma esfera ideal (modelada como um sistema de partículas) rolando sem deslizamento sobre um plano inclinado ideal não realiza trabalho. Se a força de atrito estático realizasse algum trabalho, não teríamos a igualdade (4): haveria um termo a mais no membro esquerdo da mesma. Adicionalmente, se a força de atrito estático, além de realizar trabalho, fosse conservativa, tal termo extra corresponderia a uma forma de energia potencial, associada ao atrito estático. Novamente: uma força que não realiza trabalho sobre um corpo não pode alterar sua energia mecânica, mas de forma alguma isso necessariamente implica que ela seja uma força conservativa. Mostramos, na seção anterior, que a força de atrito estático não pode ser classificada como conservativa. Também mostramos, indiretamente, que não é correto afirmar, de forma generalizada, que a força de atrito estático não realiza trabalho, ou que ela não altera a energia mecânica de um corpo. Infelizmente, no artigo de Silva e colaboradores houve um equívoco não só quanto ao conceito de força conservativa, mas também quanto à possibilidade de que uma força de atrito estático modifique a energia mecânica de um corpo. Os autores analisaram um sistema físico específico (esfera rolando sobre um plano inclinado) e extraíram conclusões que não têm validade geral.

Queremos ressaltar, no entanto, que, em nossa opinião, há muitos aspectos positivos no artigo de Silva e colaboradores, e recomendamos sua leitura - com as ressalvas que fizemos aqui.

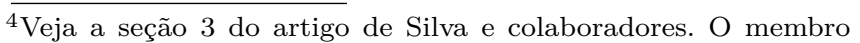
esquerdo e o membro direito da igualdade (4), acima, correspondem, respectivamente, à igualdade (3) e à igualdade (5) daquele artigo, com $m$ no lugar de $M, h$ no lugar de $|\Delta h|$ e $V_{C M}$ no lugar de $v_{\mathrm{cm}}$. (Podemos, é claro, eliminar " $M$ " em ambos os membros da igualdade 4.)
}

\section{Conclusão}

Mostramos que a força de atrito estático não pode ser classificada como conservativa. Também mostramos que não é correto afirmar, de forma generalizada, que a força de atrito estático não realiza trabalho, ou que ela não altera a energia mecânica de um corpo.

\section{Agradecimento}

Agradecemos ao professor Armando Menezes por nos ter apresentado o artigo [1] que deu origem a este trabalho.

\section{Material suplementar}

O seguinte material suplementar está disponível online: Apêndice: trabalho total das forças internas em um corpo rígido

\section{Referências}

[1] W.P. Silva, C.M.D.P.S. Silva, J.W. Precker, D.D.P.S Silva, I.B. Soares e C.D.P.S. Silva, Revista Brasileira de Ensino de Física 25, 4 (2003).

[2] D. Halliday, R. Resnick e J. Walker, Fundamentos de Física (LTC, Rio de Janeiro, 2012), v. 1, $9^{\mathrm{a}}$ ed.

[3] P.A. Tipler e G. Mosca, Física para Cientistas e Engenheiros (LTC, Rio de Janeiro, 2013), v. 1, $6^{\text {a }}$ ed.

[4] H.M. Nussenzveig, Curso de Física Básica (Edgard Blücher, São Paulo, 2002), v. 1, 5 $5^{\mathrm{a}}$ ed.

[5] M. Alonso e E.J. Finn, Física, um Curso Universitário (Edgard Blücher, São Paulo, 1972), v. 1.

[6] H.D. Young e R.A. Freedman, Física (Pearson Education do Brasil, São Paulo, 2009), v. 1, $12^{\mathrm{a}}$ ed.

[7] A. Chaves, Física Básica (LTC, Rio de Janeiro, 2012), v. 1.

[8] R.P. Feynman, R.B. Leighton e M. Sands, Lições de Física de Feynman (Bookman, Porto Alegre, 2008), v. 1.

[9] J.R. Taylor, Mecânica Clássica (Bookman, Porto Alegre, 2013).

[10] S.T. Thornton e J.B. Marion, Dinâmica Clássica de Partículas e Sistemas (Cengage Learning, São Paulo, 2011), $5^{\mathrm{a}}$ ed.

[11] H. Goldstein, C. Poole e J. Safko, Classical Mechanics (Addison Wesley, São Francisco, 2002), $3^{\text {a }}$ ed. 